\title{
Improvement of bias detection in Argo float conductivity sensors and its application in the North Atlantic
}

\author{
Cabanes Cécile ${ }^{1,2,{ }^{*}}$, Thierry Virginie ${ }^{3}$, Lagadec Catherine ${ }^{3}$
}

${ }^{1}$ Univ. Brest, CNRS, IRD, Ifremer, Laboratoire d'Océanographie Physique et Spatiale (LOPS), IUEM, Brest, France

${ }^{2}$ Univ. Brest, CNRS, IRD, Unité Mixte de Service 3113, IUEM, Brest, France

${ }^{3}$ Ifremer, Univ. Brest, CNRS, IRD, Laboratoire d'Océanographie Physique et Spatiale (LOPS), IUEM, Brest, France

*Corresponding author : Cécile Cabanes, email address : $\underline{\text { Cecile.Cabanes@ifremer.fr }}$

\begin{abstract}
:
We propose modifications of the OW method (Owens and Wong, 2009) used to estimate the timevarying correction of conductivity measurements from Argo floats. These modifications are necessary to account for large interannual to decadal variability of the large-scale salinity field observed, for instance, in the North-Atlantic Ocean and to provide corrections with realistic error bars. The covariance function used to map reference salinity data at the float profile position was modified in order to minimize the contribution of the oldest reference data to the large-scale salinity field estimate. Mapping error now includes errors in the large-scale field estimates and fit error now takes into account the lateral dependence between climatological profiles. Finally, we used the modified OW method to check the consistency of the Argo salinity dataset available in delayed mode in the North-Atlantic Ocean. Overall, salinity corrections need to be reconsidered for $4.5 \%$ of the floats.
\end{abstract}

\section{Highlights}

Method used to detect bias in Argo float conductivity sensors is improved $>$ Temporal variability of the large-scale salinity field is taken into account Computation of the errors for salinity data corrections is improved Consistency of the salinity data corrections in the North Atlantic is checked

\section{Introduction}

The primary objective of the Argo project (http://www.argo.ucsd.edu/) is to document the current and changing state of the upper ocean, including heat and freshwater storage and transport. To achieve this objective, Argo target accuracies for measurements are $5 \mathrm{~dB}$ for pressure, $0.005^{\circ} \mathrm{C}$ for temperature, and 0.01 for salinity (Argo Science Team, 2000). Although temperature and pressure measurements may be subject to instrument errors or failures (Willis et al., 2007; Barker et al., 2011), these parameters are generally measured within the required 
accuracies throughout the life of a float. Salinity measurement is more problematic due to sensitivity to the inductive field in the formerly-used FSI sensors or to the geometry of the conductivity cell in the currently-used Seabird sensors (SBE41). As it is necessary to check salinity data for drift or offset of the conductivity sensor, efforts have been made since the beginning of the Argo project to standardize delayed mode procedures for salinity correction. These procedures are regularly updated (Argo quality control manual Version 2.9.1, Wong et al., 2014).

Salinity data are checked in delayed mode, at least one year after float deployment, through the combined use of statistical tools and scientific expertise. The most common method is based on the comparison of Argo salinity profiles with calibrated reference measurements. The OW software (Owens and Wong, 2009) uses this method and has been made freely available to the Argo community, which uses it widely because it can be applied to float drifting anywhere in the global ocean. The OW method improved on the method originally developed by Wong et al. (2003) and also took into account the modifications proposed by Böhme and Send (2005) for highly variable environments and for regions where the flow is bathymetrically controlled, such as the Subpolar North Atlantic. The reference dataset used for this comparison is made up primarily of high-quality shipboard CTD data from research cruises, and is supplemented by the more comprehensive dataset of previously-verified Argo data.

Although the OW method was designed to minimize manual and subjective choices, scientific expertise is necessary to decide whether the conductivity sensor has actually experienced a problem. Indeed, ocean variability can sometimes be misinterpreted as a sensor drift or offset. This scientific expertise requires a good knowledge of the behaviour of the float, of the sensor, of the water masses sampled and of their variability, and often necessitates the analysis of other observations (e.g. hydrographic profiles acquired at float deployment). Generally, it is the Principal Investigator (PI) of the float who makes the final decision on whether to correct the float salinity data or not. Whenever a salinity drift or offset is confirmed, the correction provided by the OW method is applied.

An additional review of basin-wide data quality is regularly performed at the Argo Regional Centres (ARCs) level. One of the objectives of the ARCs is to ensure that the delayed mode salinity corrections are consistent within a basin. These checks are necessary because delayed mode analyses are conducted by different operators from different national data centres and 
because procedures, methods, and reference datasets have evolved since the beginning of the Argo project.

The North Atlantic ARC (NA-ARC), which is one of the five ARCs, is in charge of all the float data in the North Atlantic north of $20^{\circ} \mathrm{S}$ (the tropical band $20^{\circ} \mathrm{S}-20^{\circ} \mathrm{N}$ is shared with the South Atlantic ARC). As part of the NA-ARC activities, we wanted to check the consistency of the delayed mode salinity data in the North Atlantic. Before making this assessment, we selected all unbiased floats in the North Atlantic and checked whether the OW method run with the same standard settings for all these floats gave results that generally agreed with the PI's decision (i.e. that no correction was necessary). We found that the OW method suggested that the salinity measured by many floats was either too high in the Subpolar North Atlantic or too low in the Western Subtropical Atlantic. This means that the PIs ${ }^{e e}$ decision not to correct these floats was not based on the results of the OW method, but on other observations, like comparison with shipboard CTD profiles made at float deployment for instance. We thus investigated why we observed this apparent contradiction. Although fine tuning of the OW method for a particular float may have helped to avoid ambiguity, we found that some modifications of the OW method were necessary to better take into account the large decadal/ interannual variability of the deep water masses and to provide more realistic error bars.

The objective of this paper is to detail those modifications and to assess the consistency of the Argo delayed mode salinity data in the North Atlantic. Section 2 presents the dataset used in this study and the OW method. Section 3 evaluates the OW method in the North Atlantic. Section 4 describes the modifications to the OW method and presents the results of its application to North-Atlantic floats for which delayed mode data is available, including those for which a salinity drift or offset was detected by the floats ${ }^{\text {ee }}$ PI. Finally, Section 5 concludes the paper.

\section{Data and Method}

\section{Argo data}

In this study, a snapshot of Argo data available on the Global Data Centre server as of 25/07/2013 was used. We selected all the unbiased floats available in the North-Atlantic region (north of $30^{\circ} \mathrm{N}$ ) at the date of the analysis (i.e. those for which no salinity drift or offset was identified during the delayed mode checks performed by the PI of the float). To identify 
this set of floats, the vertical mean difference between the raw salinity profile (PSAL) and the adjusted salinity profile obtained in delayed mode (PSAL ADJUSTED) was calculated. In cases where no salinity correction was necessary, the adjusted profile is simply equal to the raw profile. Therefore, a float was selected when the mean (PSAL - PSAL ADJUSTED) over depth was lower than 0.002 for all of its salinity profiles. The small tolerance of 0.002 was set to allow the selection of floats that had a raw pressure adjustment that was typically less than $5 \mathrm{dbar}$, and for which the adjusted salinity was computed in accordance with the adjusted pressure but with no further salinity corrections. All the SOLO floats with FSI sensors were excluded because they are known to have pressure issues (Willis et al., 2007). Finally, 392 floats were selected in the North Atlantic. These unbiased floats were used to evaluate the OW method in this region.

\section{The OW method}

The OW method is based on an objective analysis method (Bretherton et al., 1976). The details of this method can be found in Owens and Wong (2009), Wong et al. (2003) or Böhme and Send (2005). The three main steps of the method are given below, focussing on what is essential to understand the modifications proposed in section 3 of the present paper.

1. For each profile at $\left(x_{0}, y_{0}, t_{0}\right)$ of a given float, a set of reference salinity profiles at $\left(x_{i}, y_{i}, t_{i}\right)$ is selected. The objective of the selection is to retain the reference profiles that are closest positioned and most contemporaneous to the float profile date, as well as those with measurements obtained on the same isobaths as the float profile. This is particularly important in regions where the water mass distribution is strongly controlled by bathymetry, as in the North Atlantic. The selection criteria are based on the computation of ,separation factors ${ }^{\text {ee }}$ (see Wong et al., 2003), which are relative, first, to pre-defined large length scales ( $L_{x}$ and $\left.L_{y}\right)$ and a large cross-isobath scale $(\Phi)$, and, then, to pre-defined small length scales $\left(1_{\mathrm{x}}\right.$ and $\left.1_{\mathrm{y}}\right)$, a small cross-isobath scale $(v)$ and a temporal scale $\tau$.

A maximum of $\mathrm{N}$ profiles are selected among all the reference profiles available within an area that extends over three times the large spatial scales and is defined by:

$$
\frac{\left(x_{i}-x_{0}\right)^{2}}{\left(3 L_{x}\right)^{2}}+\frac{\left(y_{i}-y_{0}\right)^{2}}{\left(3 L_{y}\right)^{2}}+\frac{\left(q_{i}-q_{o}\right)^{2} /\left(q_{i}^{2}+q_{0}^{2}\right)}{\Phi^{2}}<1
$$

where $\mathrm{q}$ is the barotropic potential vorticity and the third term of Eq. (1) accounts for the cross-isobath separation. First, N/3 profiles are randomly selected within the area defined by 
Eq. (1) to ensure that the large-scale mean is well represented. From the remaining profiles, $\mathrm{N} / 3$ profiles with the shortest separation factor relative to the large scales $L_{x}, L_{y}$ and $\Phi$ are selected. Finally, again from the remaining profiles, N/3 profiles with the shortest separation factor relative to small scales $l_{x}, l_{y}$ and $v$, and to the temporal scale $\tau$ are selected. The set of selected reference salinity data is denoted $d=\left[d_{1}, \ldots, d_{m}\right]$, with $m \leq N$.

2. For each reference profile selected during the first step, salinity data are vertically interpolated onto the potential temperatures measured by the float (float profile $\theta$ levels). Salinity of the reference profiles is then objectively mapped at the float profile location using a two-stage optimal interpolation (OI) process based on Roemmich (1983). First, a large-scale field $S^{\prime}$ is estimated on each float profile $\theta$ levels, using the set of reference salinities:

$$
S^{\prime}=\langle d\rangle+W \cdot(d-\langle d\rangle)
$$

where $\langle d\rangle$ is a mean salinity field obtained within the area defined by Eq. 1 from the $\mathrm{N}$ profiles selected at step 1, and where the weighting matrix $W=C d m \times C d d^{-1}$ is the product of the data-mapping point covariance matrix $C d m$ and the inverse of the data-data covariance matrix. It is assumed that the large-scale field $S^{\prime}$ is time-independent and that the covariance matrices $C d m$ and $C d d$ have a Gaussian shape with a decay determined only by the large spatial scales $\left(L_{x}\right.$ and $\left.L_{y}\right)$ and the large cross-isobath scale $(\Phi)$. The large-scale field is also estimated at each reference data position and subtracted from the reference salinity data. Secondly, the residuals $r=\left[r_{1}, \ldots, r_{m}\right]$ obtained, are used to estimate the small-scale field $S^{\prime \prime}$. In this case, the Gaussian decay for the covariance matrices is determined by the temporal scale $\tau$, the small spatial scales $\left(1_{\mathrm{x}}\right.$ and $\left.1_{\mathrm{y}}\right)$ and small cross-isobath scale $(v)$. The objectively mapped salinity is finally obtained from the sum of the large-scale estimate $S^{\prime}$ and the smallscale estimate $S^{\prime \prime}$ :

$$
S_{\text {mapped }}=S^{\prime}+S^{\prime \prime}
$$

The OI process also provides errors for the objectively mapped salinity. The mapping error $\sigma$ is determined from the second stage of the mapping and is large if the signal variance of the residuals $r$ is large and if contemporaneous data are not available or were obtained at a large distance from the float position relative to the small spatial scales and cross-isobath scales. 


\section{Considering all the profiles of a given float, a time-varying potential conductivity} correction is estimated as explained below. It is assumed that the potential conductivity correction for a given profile takes the form of a multiplicative factor that is similar to a constant salinity offset over depth. The time-varying multiplicative factor is estimated using a calibration model that adjusts the float potential conductivity measurements to the objectively mapped potential conductivities within stable water masses. Indeed, only the ten most stable $\theta$ levels are used, namely $\theta$ surfaces in the float time series that have the minimum salinity variance on $\theta$ levels. The calibration model, which consists of a set of straight-line fits between breakpoints, is weighted by the inverse of the mapping error variance. This means that objectively mapped salinities (and therefore potential conductivities) with large mapping errors have a reduced weight for the calibration. The optimal number of breakpoints and their position in the float time series are objectively determined by a statistical method. If needed, the user can also impose the number of breakpoints. The additive adjustment in salinity $\left(\Delta S_{f i t}\right)$ is then obtained from the multiplicative factor used to adjust the float potential conductivity. The method provides a statistical uncertainty for the fit (fit error) assuming vertical correlation between data points ( $\theta$ levels) but lateral independency. The error covariance matrix is:

$$
R^{2}=\operatorname{diag}\left(\sigma^{2}(c)\right) \times L_{v c o v}
$$

where $\sigma(c)$ is the mapping error for conductivity and $L_{v c o v}$ is the vertical covariance matrix. Vertical scales are estimated using the vertical extents of water masses that are inferred from a generic vertical water mass structure of a typical ocean basin (see Wong et al., 2003). If the PI considers that sensor malfunction has occurred, it is recommended to make an adjustment of float salinity data as soon as $\Delta S_{f i t}$ is greater than twice the fit error and greater than the instrument accuracy, which is 0.01 (Argo quality control manual, Wong et al., 2014).

Finally, diagnostic plots are produced. As an example, two of these diagnostic plots are shown in Figures $1 \mathrm{a}$ and $1 \mathrm{~b}$ for the float 5902269. Figure 1a shows the float path with the bathymetry. The float 5902269 was deployed in the Irminger Sea, southeast of Greenland, in June 2010. It then drifted at 1000 meters depth following the main deep currents and reached the Labrador Sea, southwest of Greenland. Figure $1 \mathrm{~b}$ shows the time series of $\overline{\Delta S_{\text {sta }}}$ and $\Delta S_{f i t}$ as a function of the float cycle number, where $\overline{\Delta S_{\text {sta }}}$ is the difference between mapped salinities and float salinities vertically-averaged on the ten $\theta$ levels, and $\Delta S_{f i t}$ is the additive adjustment in salinity. The difference $\overline{\Delta S_{s t a}}$ is most often negative, reaching -0.03 near cycle 
100. Here, a constant - over time-multiplicative adjustment in conductivity was imposed; therefore the additive adjustment in salinity is also very close to a constant.
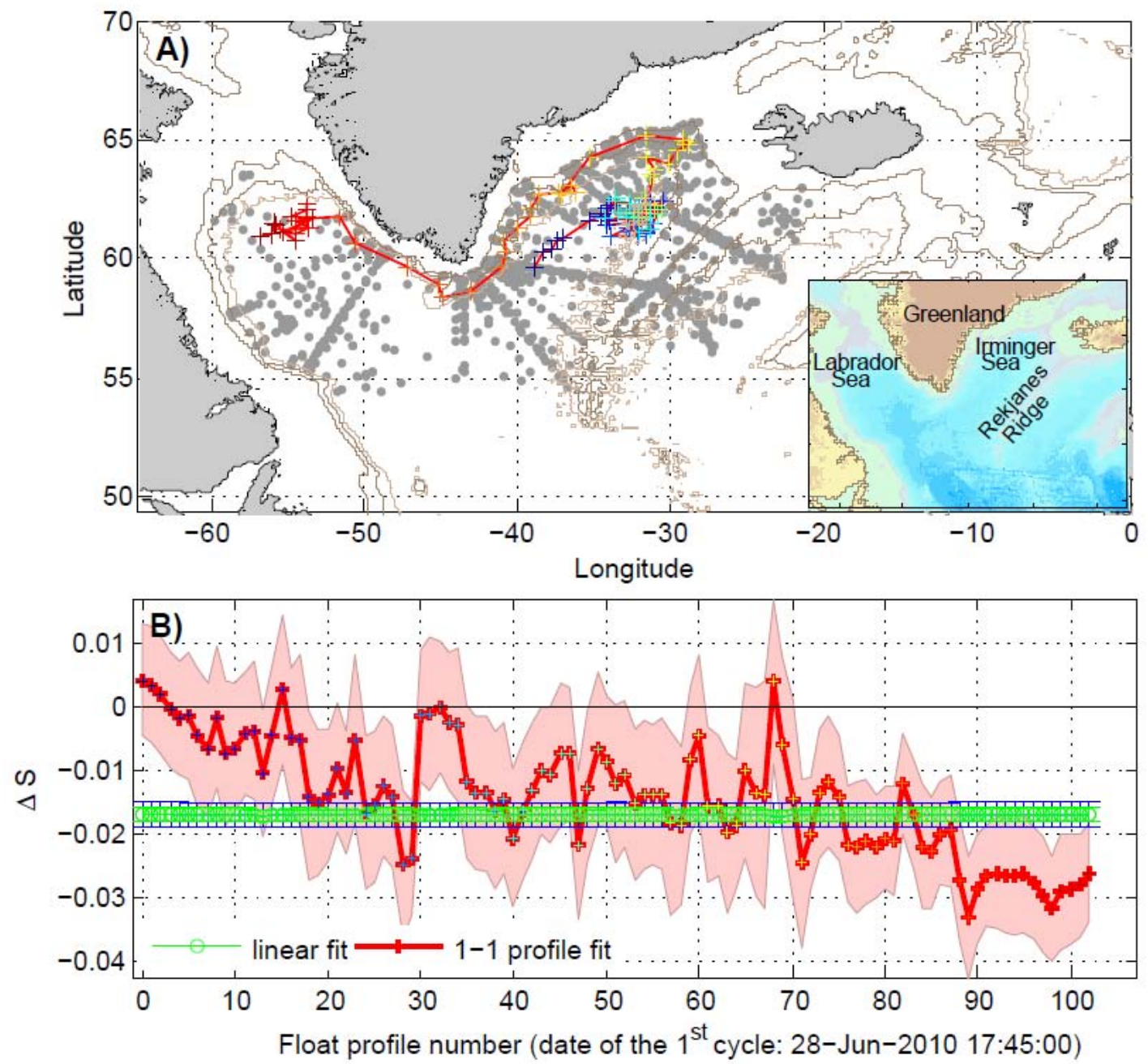

Figure 1: Results of the OW method for the float 5902269 using the shipboard CTD reference database. (A) Shipboard reference CTD profiles used for the mapping (grey dots) are shown on the map along with the float trajectory (coloured crosses). (B) Vertically-averaged mapped salinities minus float salinities on the ten most stable $\theta$ levels (red lines and coloured crosses) and the offset obtained by the linear fit (green circles). The mapping errors are shaded in red. Green error bars show the fit error and blue error bars show the doubled fit error. The colour of the crosses on panels (A) and (B) correspond to the profile number, from the first profile (black-blue) to the last profile (red).

\section{Reference databases}

Together with the OW method, two reference databases are supplied to the delayed mode operators and regularly updated by the Argo program. The first of these databases contains 
historical shipboard CTD data obtained from the World Ocean Database, from the CLIVAR and Carbon Hydrographic Data Office (CCHDO), or directly from individual scientists. The second database contains historical Argo profiles with no salinity adjustment in delayed time. For this study, we used the reference CTD database (version 2013v01) and Argo database (version 2013V01) updated in 2013.

\section{Evaluation of the OW method in the North-Atlantic region}

The OW method was run for each of the 392 unbiased floats, using the historical shipboard CTD reference database only or both historical shipboard CTD and Argo reference databases (CTD+Argo hereafter). Following Böhme and Send (2005), specific settings of the OW method parameters for the North-Atlantic region (see table 1) were applied. Because most of the 392 floats considered here were also present in the Argo reference database, the data from the float processed with the OW method were excluded from the Argo reference database.

Table 1: Configuration parameters used in this study to run the OW method and the modified OW method.

\begin{tabular}{lll}
\hline $\begin{array}{l}\text { PARAMETER } \\
\text { (units) }\end{array}$ & $\begin{array}{l}\text { VALUE } \\
\text { OW method }\end{array}$ & $\begin{array}{l}\text { VALUE } \\
\text { modified OW } \\
\text { method }\end{array}$ \\
\hline $\mathrm{N}$ & 250 & 250 \\
$L_{x}\left({ }^{\circ}\right)$ & 3.2 & 3.2 \\
$l_{x}\left({ }^{\circ}\right)$ & 0.8 & 0.8 \\
$L_{y}\left({ }^{\circ}\right)$ & 2 & 2 \\
$l_{y}\left({ }^{\circ}\right)$ & 0.5 & 0.5 \\
$\Phi$ & 0.1 & 0.1 \\
$\phi$ & 0.02 & 0.02 \\
$\tau(\mathrm{yr})$ & 0.69 & 0.69 \\
$\mathrm{~T}(\mathrm{yr})$ & - & 2 \\
\hline
\end{tabular}

For each profile of each float, the difference between mapped salinities and float salinities averaged over the ten most stable $\theta$ levels $\left(\overline{\Delta S_{s t a}}\right)$ was computed. The values of $\overline{\Delta S_{s t a}}$ are displayed at the float profile positions (Figures $2 \mathrm{a}$ and $2 \mathrm{~b}$ ). One would expect that the differences obtained for the unbiased floats would be distributed around zero. However, 
systematic negative differences were found in the Subpolar Gyre, particularly along the Reykjanes Ridge and the topography of the Labrador Sea, and systematic positive differences were found in the western part of the Subtropical Gyre. These systematic differences were clearly evident when float measurements were compared with the historical shipboard CTD reference database (Figure 2a). Using the more recent CTD+Argo reference database did not change the regional patterns, although it reduced the amplitude of the differences between mapped salinities and float salinities (Figure 2b).
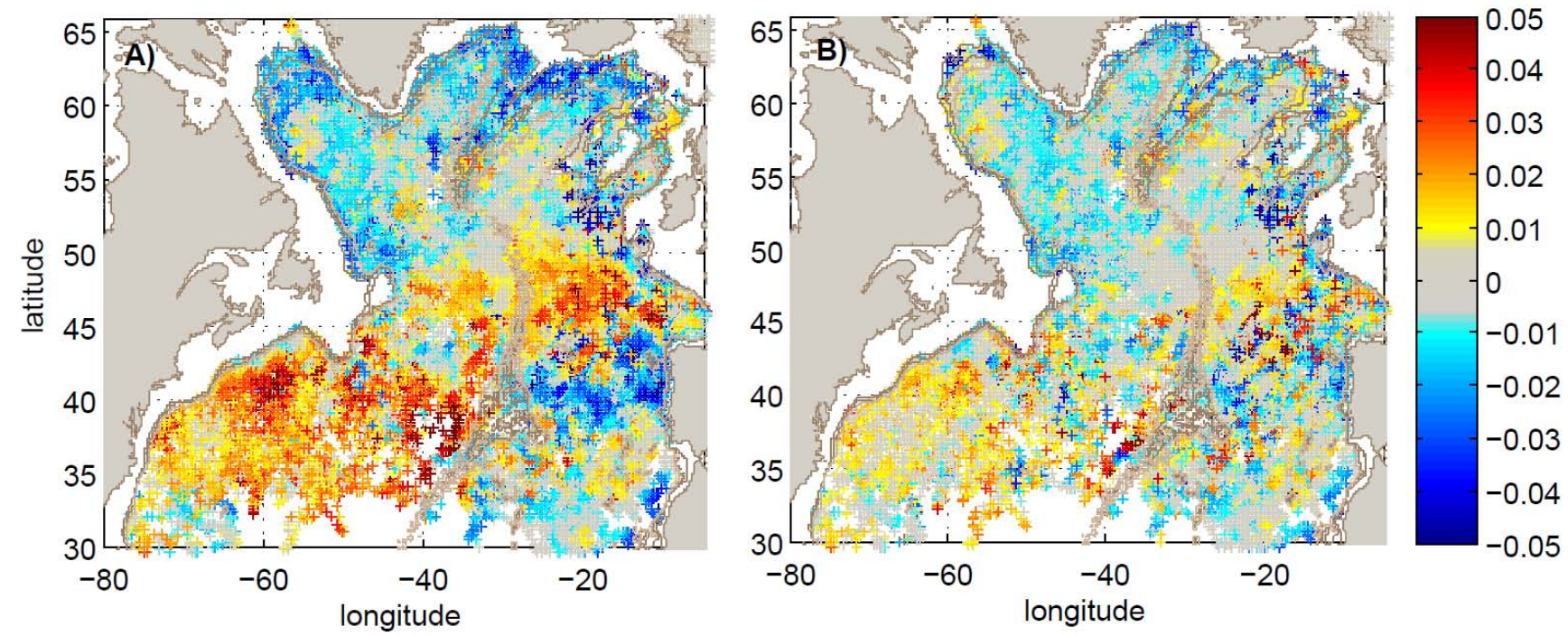

Figure 2: Differences between mapped salinities and float salinities averaged over $10 \theta$ levels of a profile. A) shipboard CTD reference database used alone. B) CTD +Argo reference databases used together.

For each float, a constant salinity offset over time $\Delta S_{\text {fit }}$ was estimated from the fit of mapped conductivities and float conductivities. A constant offset over time is not necessarily the best fit in a statistical sense for all of the 392 floats, but it gives a first indication on how well the float salinities match the mapped salinities. Figure 3 shows the distribution of the salinity offsets for all the floats. 

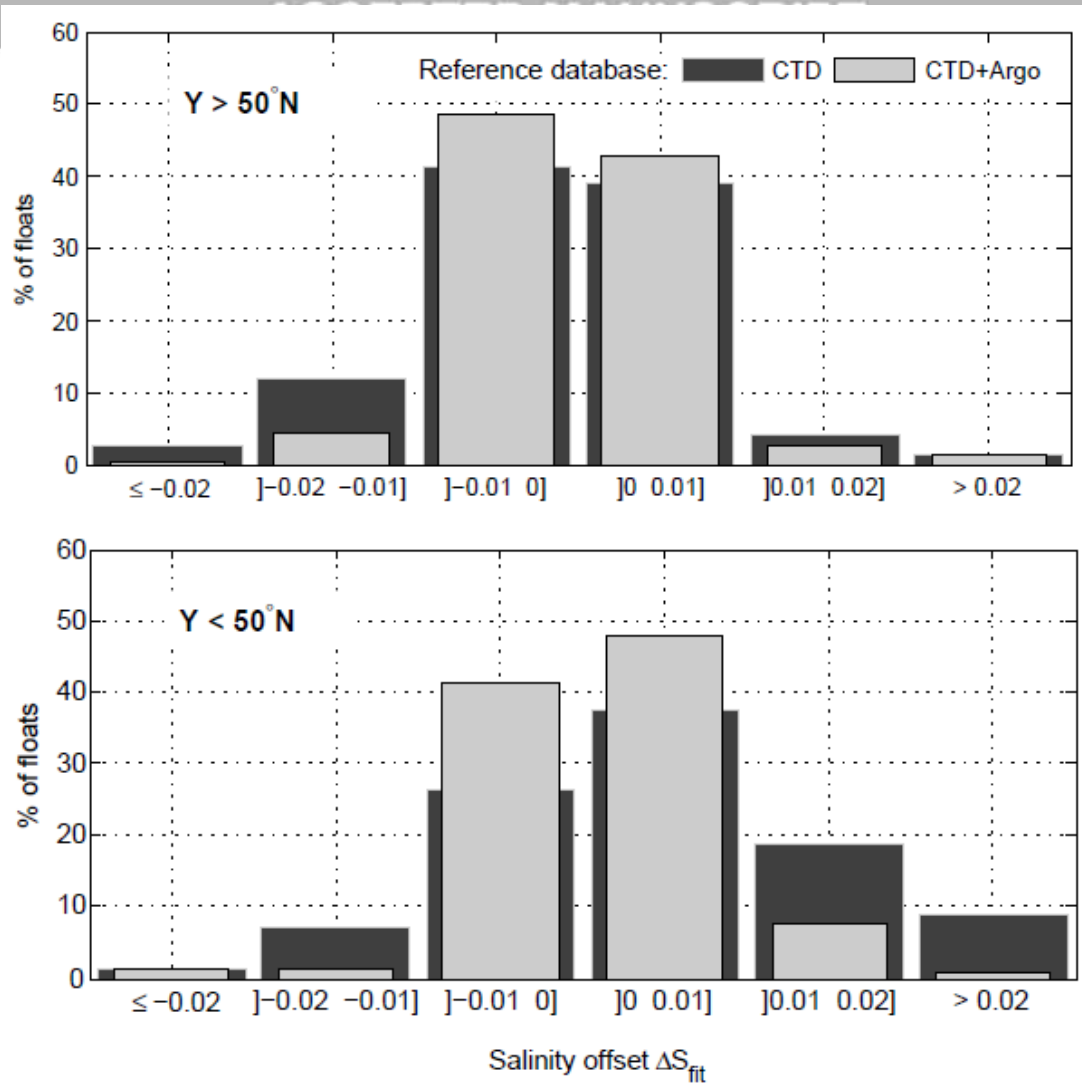

Figure 3: Distribution of the salinity offsets obtained when the OW method was run with the shipboard CTD reference database (dark grey) and the CTD+Argo reference database (light grey) on the 160 floats found north of $50^{\circ} \mathrm{N}$ (upper panel) and on the 232 floats found south of $50^{\circ} \mathrm{N}$ (lower panel) in the North-Atlantic Ocean.

As expected from the values of $\overline{\Delta S_{\text {sta }}}$ negative salinity offsets were more often obtained in the Subpolar Region, north of $50^{\circ} \mathrm{N}$ and positive salinity offsets were more often obtained south of $50^{\circ} \mathrm{N}$, although negative salinity offsets were also found here, mainly in the eastern part of the basin. When the OW method was run with the shipboard CTD reference database alone, salinity offsets were lower than -0.01 for about $15 \%$ of the floats north of $50^{\circ} \mathrm{N}$ and greater than 0.01 for about $20 \%$ of the floats south of $50^{\circ} \mathrm{N}$. These results are in contradiction with the $\mathrm{PI}^{\mathrm{Ie}} \mathrm{s}$ decision not to correct the float data. Using the more recent CTD+Argo reference database reduced the amplitude of the estimated offsets (see Figure 3), but offsets obtained for about 40 floats were still above the recommended threshold to make a salinity adjustment (i.e. above the instrument accuracy (0.01) and above twice the fit error). The fit errors were indeed very low (less than 0.0025 for $92 \%$ of the offsets). 


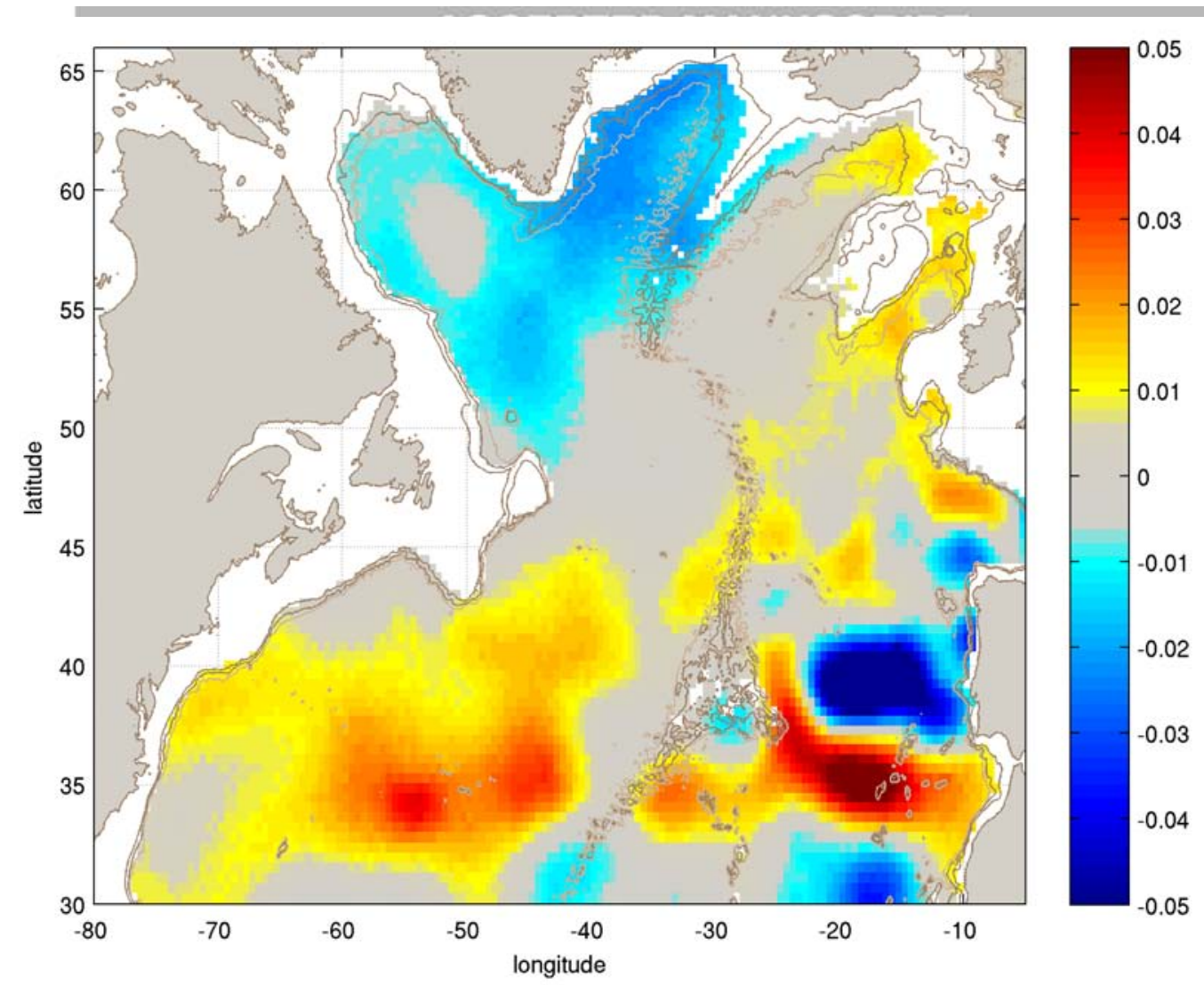

Figure 4: Salinity difference at 1500m depth between the WOA05 climatology and the monthly salinity fields produced by the ISAS-13 analysis and averaged over the period 2004-2012. Note that the colour bar matches the one in Figure 2: negative (positive) values indicate that the ISAS-13 climatology is saltier (fresher) than the WOA05 climatology.

There are two possibilities that could explain the differences between the OW results and the $\mathrm{PI}^{\mathrm{ee}} \mathrm{s}$ decision. Either the PIs were right not to correct the float salinity data, which means that the amplitude of the offsets obtained with the OW method were too large for a significant number of floats and/or that the fit errors were too low. Or the PIs were wrong, which means that salinity data of the Argo floats were systematically biased toward too salty values in the Subpolar North Atlantic and toward too fresh values in the Western Suptropical gyre. This hypothesis seems unrealistic as we cannot see any reason that would explain such a spatial pattern with systematic fresh bias in one region and salty bias in another. We thus hypothesized that this pattern mainly came from the OW method, which failed to reproduce the decision of the PI.

Interestingly, the spatial pattern of the difference between the mapped salinities and the float salinities (Figure 2a) is similar to that of the decadal salinity changes observed at depth in the 
North Atlantic (Figure 4). Indeed, we compared the monthly salinity fields produced by the ISAS analysis (In Situ Analysis System, Gaillard et al., 2009) and averaged over the period 2004-2012 (ISAS-13, Gaillard, 2015) with the WOA05 climatology (Antonov et al., 2006). ISAS uses the OI method to produce gridded fields of temperature and salinity data. Most of the data used for the ISAS-13 analysis come from Argo floats. The fields used to generate WOA05 climatological maps were computed by objective analysis of all scientifically quality-controlled historical salinity data in the World Ocean Database 2005 (Boyer et al., 2005). The difference between the WOA05 salinity climatology and ISAS-13 mostly reflects salinity changes between the pre-2005 period and 2004-2012. Salinity changes at 1500 metredepth are mainly broad-scale changes with an increase of salinity in the Subpolar Gyre and a decrease of salinity in the western subtropical gyre (Figure 4). Similar salinity changes were also observed along repeated hydrographic sections in the North Atlantic. For example, four occupations of the repeated zonal transatlantic section along $59.5-60^{\circ} \mathrm{N}$ (from the Scottish shelf to Cape Farewell) showed that the deep Labrador Sea Water (dLSW), which was found around $1500 \mathrm{~m}$ in the Irminger sea, became saltier by 0.04 on average between 1997 and 2006 (Sarafanov et al., 2007). In the subtropical gyre, Leadbetter et al. (2007) showed that intermediate waters at $36^{\circ} \mathrm{N}$ became cooler and fresher between 1981 and 2005. Particularly, the Labrador Sea Water observed at $55^{\circ}-65^{\circ} \mathrm{W}$ and $1500-2500 \mathrm{~m}$ became fresher by $0.01-0.03$. These observations, based only on shipboard CTD data, support the hypothesis that the largescale variability shown in Figure 4 is linked to interannual to decadal changes in water mass properties and not erroneously induced by systematic bias in Argo floats.

Considering that the comparisons between float data and reference data were generally done on $\theta$ levels between 1000 and $2000 \mathrm{~m}$, the aforementioned similarity suggests that the mapped salinities are relaxed back toward a climatological large-scale field that does not reflect thermohaline properties at the time the Argo profile was collected. This is further supported by the fact that using Argo data in the reference database (and hence more recent data) reduces the amplitude of the observed spatial pattern (Figure 2b). As the climatological largescale field is significantly fresher (saltier) than the salinity measured more recently by a float in the Subpolar (Subtropical) Gyre (Figure 4), the additive correction $\left(\overline{\Delta S_{\text {sta }}}\right)$ proposed for each profile tends to be negative in the Subpolar North Atlantic and positive in the western subtropical region. 
To make a more detailed examination, we studied the case of float 5902269. As shown in

Figure $1 \mathrm{~b}$, negative values of $\overline{\Delta S_{\text {sta }}}$ were found for this float along the topography of the Labrador Sea. Figure 5 shows one of the profiles (profile 90) measured by this float near $50^{\circ} \mathrm{W}$ in the Labrador Sea, the corresponding mapped profile, and all the reference profiles from the Argo reference database used for the mapping. Reference profiles were plotted with a colour that represents the time difference between the float measurement and the reference data. As can be seen in Figure 5, the interannual/decadal variability was quite high in this region, even at the deepest $\theta$ levels sampled by the float. The mapped salinities differed from the float profile salinities by 0.01 to 0.02 at all levels and apparently corresponded to an average of all the reference salinities, suggesting that not enough weight was given to the most recent data. This was confirmed by estimating the mapped salinity as before but excluding profiles in the reference database that were more than 2 years older or younger than the Argo profile. This mapped profile was clearly in better agreement with the float profile than the mapped profile estimated with the whole reference database.

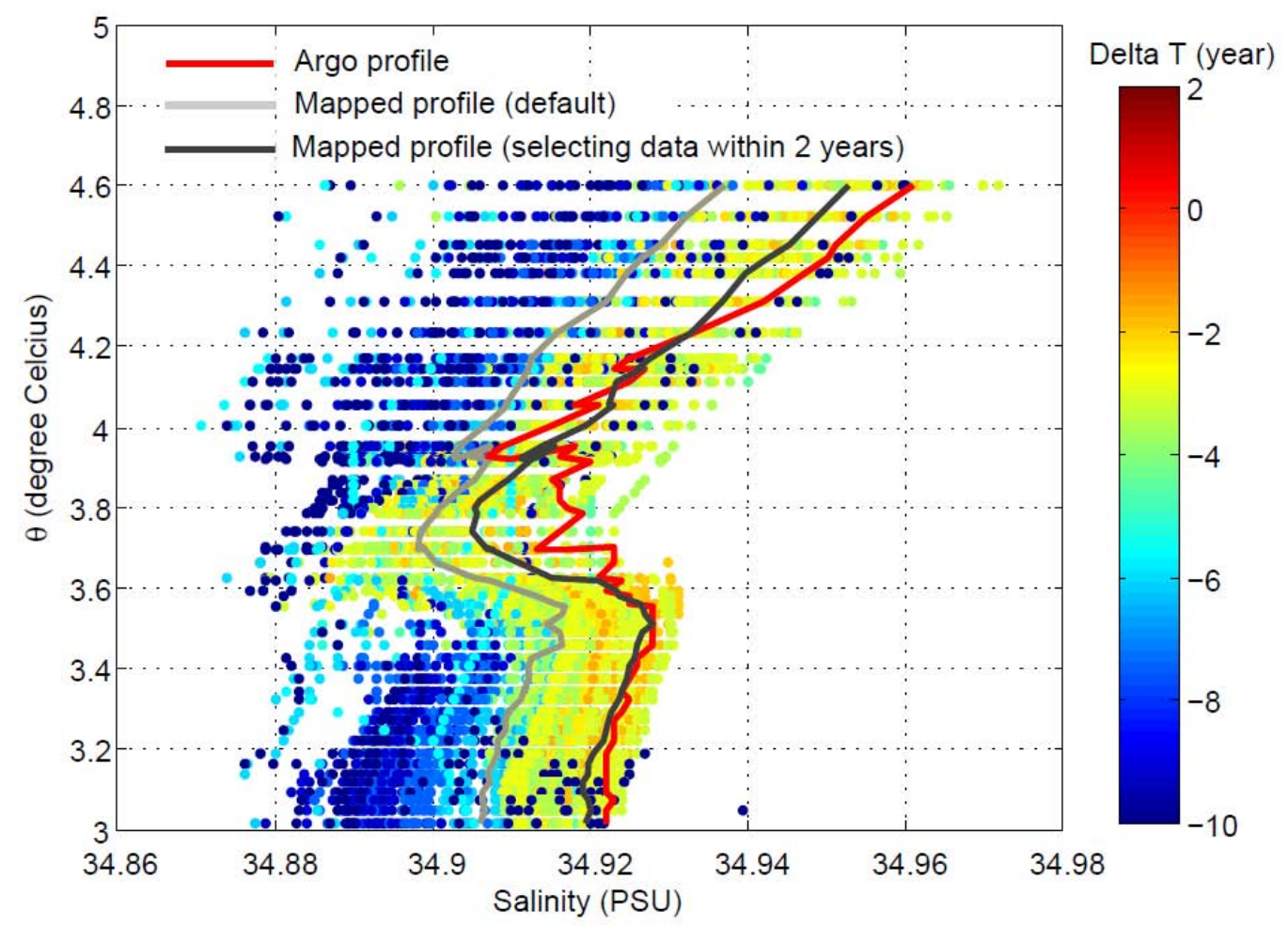

Figure 5: Profile 90 of Argo float 5902269 (red) and the mapped profile obtained using the full Argo reference database (grey), selecting data within 2 years of the float profile (black). Reference profiles used for the mapping are plotted with a colour that represents the difference Delta $T$ (in years) between the date of the Argo profile and the date of the reference profile. 
We then investigated why the mapping method did not give enough weight to the most recent data. For profile 90 of float 5902269, the contribution of the small spatial scales and recent reference profiles to the final estimate was very low compared with the contribution of the time-independent large-scale field. Indeed, the salinity estimated at the level $\theta=3.37^{\circ} \mathrm{C}$ was $S_{\text {mapped }}=S^{\prime}+S^{\prime \prime}=34.9124-0.0002$. The same was observed on the other $\theta$ levels. The most recent reference profiles had very little influence on the mapped salinity because there was not enough reference data within the small spatial scales and within the time scale $\tau$. The final mapped salinity was thus relaxed back toward the large-scale time-averaged field, as hypothesized.

To cope with this issue, we proposed modifications of the OW method to increase the contribution of recent reference data in the estimation of the large-scale salinity field and to provide more realistic fit errors, which are necessary to help PIs to decide whether or not they should apply the proposed corrections. These modifications are detailed in the next section.

\section{Modifications of the OW method and application to North-Atlantic floats}

\section{Modifications of the OW method}

We first assumed that the large-scale field $S^{\prime}$ is time-dependent with a time scale T. To account for this time dependence, one option could have been to run the OW method with the same configuration parameters as the original ones, but selecting only the reference data within $+/-\mathrm{T}$ years of each Argo profile. This option was not taken because it may lead to incorrect estimates of the offset $\Delta S_{f i t}$. For instance, we ran the OW method for the float 5902269 , selecting the reference data within $+/-2$ years of each Argo profile. Although $\overline{\Delta S_{\text {sta }}}$ values were more centred on zero, which was in better agreement with PI ${ }^{\text {ee }}$ s evaluation (i.e. no offset for this float), the offset fit $\Delta S_{\text {fit }}$ was pushed toward the negative values of $\overline{\Delta S_{\text {sta }}}$ found at the end of the floates lifetime (Figure $6 \mathrm{~b}$ ). These values were associated with the float profiles located in the Labrador Sea (see Figure 6a) for which very few reference data were available within $+/-2$ years. The signal variance, which was estimated from the few available profiles, was too low compared with the true variance expected in that area, which led to very low mapping errors. As a consequence, the offset fit, weighted by the inverse of 
the mapping error variance, was pushed toward $\bar{\Delta}$ stavalues associated with these very low mapping errors.

Instead, we chose to modify the covariance matrices $C d m$ and $C d d$ to obtain a Gaussian shape with a decay determined not only by the large spatial scales $\left(\mathrm{L}_{\mathrm{x}}\right.$ and $\left.\mathrm{L}_{\mathrm{y}}\right)$ and the large cross-isobath scale $(\Phi)$ but also by the time scale T (see Appendix A and Table 1). This increased the weight of contemporaneous data when $S^{\prime}$ was estimated.
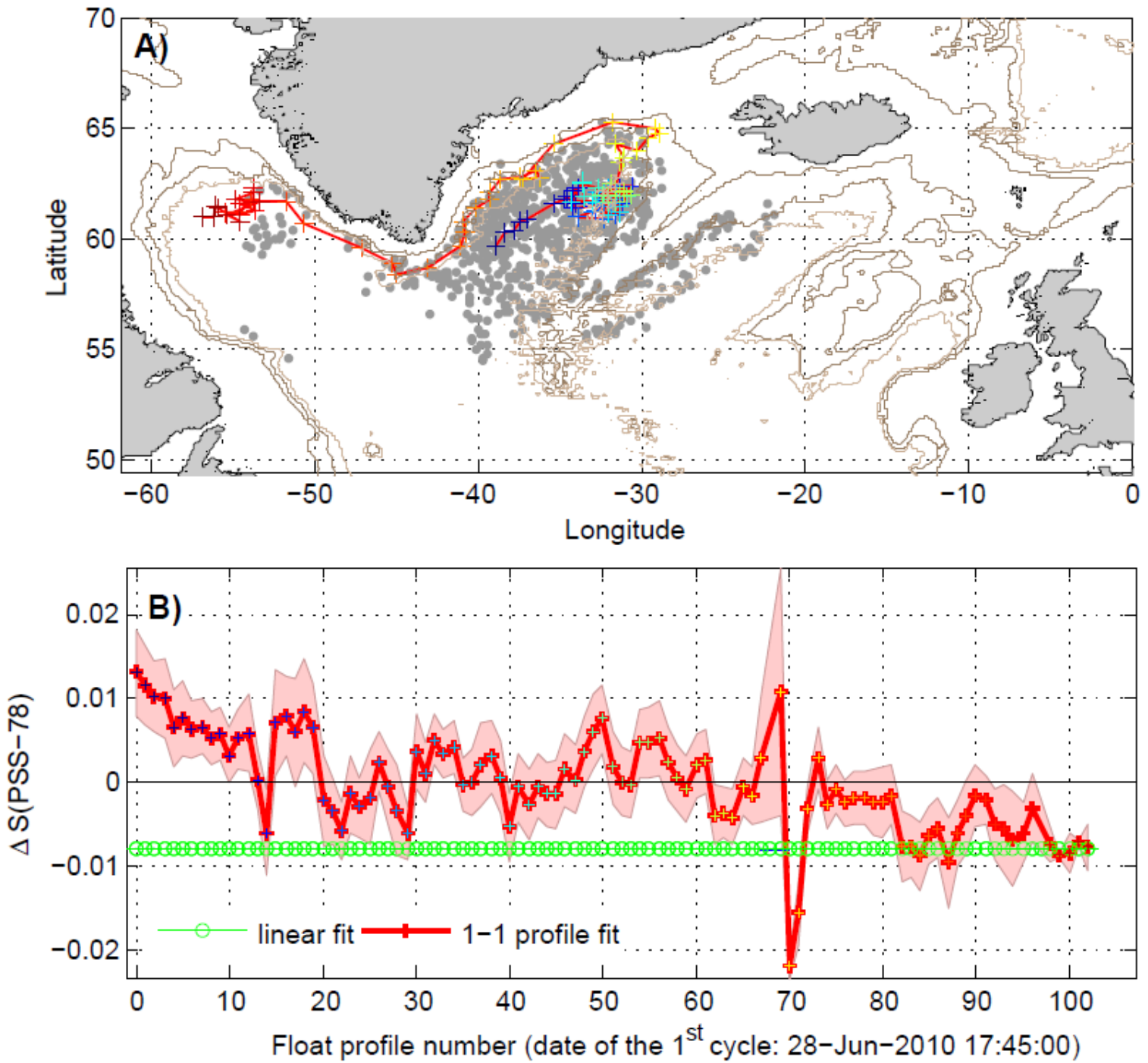

Figure 6: Same as Figure 1 but with the OW method run only with reference data (CTD+Argo) collected within 2 years of the date of the Argo profile.

If we assume that the large-scale field varies with time, it is therefore necessary to modify the estimation of the mapping errors $\sigma$. Indeed, the availability of reference data within the large spatial scale and within $+/-\mathrm{T}$ years of the Argo profile is not fully taken into account when 
the mapping errors are computed from the small-scale mapping stage only. For example, in the case where there are a lot of reference data in the vicinity of an Argo profile but acquired 10 years before the Argo profile, the 10-yr-old large-scale field will be quite well estimated at the reference data position and the residuals from the large-scale mapping stage will be small. However, if this large-scale field varies with time, it will not represent the large-scale field well at the time of the Argo profile. To take this into account, the computation of mapping errors was modified to include the errors from the large-scale mapping stage:

$$
\sigma^{2}=\sigma_{\text {large }}^{2}+\sigma_{\text {small }}^{2}
$$

where $\sigma_{\text {large }}^{2}$ and $\sigma_{\text {small }}^{2}$ are computed according to Eq. (24) in Bretherton at al. (1976), using large-scale covariance matrices and small-scale covariance matrices, respectively.

The error of the fit (model error) is based on the error covariance of the data (see Eq. 4). In this study, the error covariance matrix was constructed assuming a vertical covariance between the various $\theta$ levels and a lateral dependence between adjacent climatological profiles in the time series, as previously implemented in Wong et al. (2003). As the 10-day displacement of a float is shorter than the large spatial scales (the mean speeds in the 950$1150 \mathrm{dbar}$ layer for North-Atlantic floats was estimated at $6.7 \mathrm{~cm} \mathrm{~s}^{-1}$ by Ollitrault and Rannou (2013)), a mapped profile at an Argo profile position is built from a subset of reference profiles that is not very different from the subset used to build the mapped profile at the next or previous Argo profile position. To take into account this lateral dependency, the lateral covariance between two mapped profiles was therefore constructed using a Gaussian function with a decay determined by the large spatial scales and the large cross-isobath scale. The lateral dependency was not taken into account in Owens and Wong (2009), mainly because this can drastically reduce the number of independent samples and thus prevent the statistical determination of the optimal number of breakpoints for the piecewise linear fit. Because we chose to add a lateral covariance between adjacent climatological profiles, it is necessary to preset the number of breakpoints. This limitation can be minimised if we consider that multiple drift trends were found mainly with the first series of CTD sensors mounted on Argo floats (FSI sensors) and are no longer observed with the SBE sensors presently in use. However, if needed, it is still possible to determine the number of breakpoints automatically by first fitting the calibration time series with the lateral covariance removed, as in Owens and Wong (2009). Then, one can use the computed number of breakpoints and take into account the lateral covariance to obtain the final calibration time series. 


\section{Application to North-Atlantic floats $=$ D MANUSCRIPT}

We ran the OW method with the modifications presented above and using the CTD+Argo reference database for float 5902269. The results are shown in Figure 7.
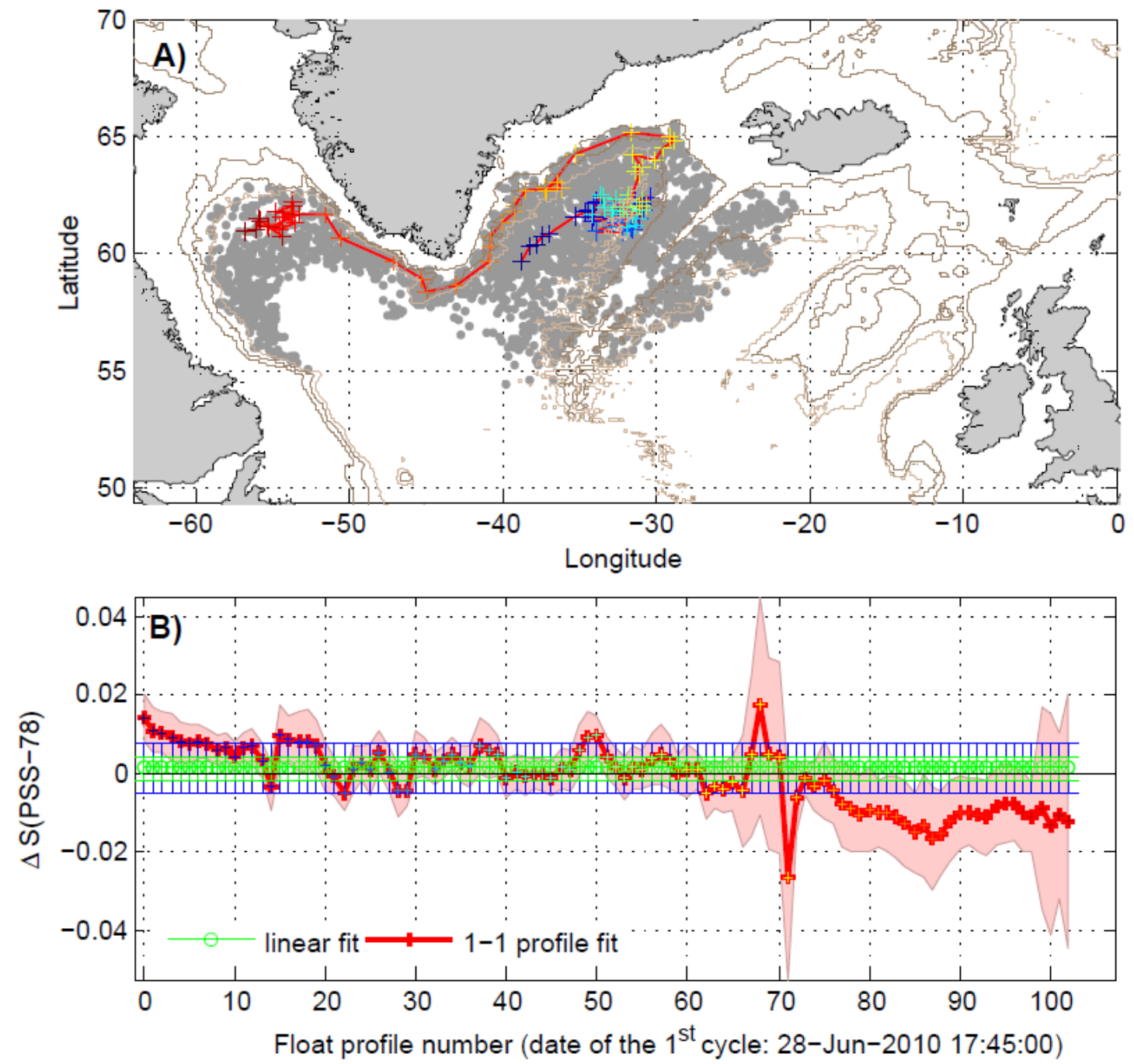

Figure 7: Same as Figure 1, but with the modified OW method run with the CTD+Argo reference database and a time scale $T$ of 2 years.

Given the observed variability in the Subpolar North Atlantic and particularly the variability of the Labrador Sea Water (e.g. Kieke and Yashayaev, 2015), we chose a relatively short time scale $\mathrm{T}$ of 2 years. After cycle 80, when the float reached the Labrador Sea, there were very few reference data within $+/-2$ years of the date of the Argo profiles (see Figure 6a). In this case, the mapped salinities were relaxed back toward the estimated mean $\langle d\rangle$ obtained within an area that spans about three times the large spatial scales (area defined by Eq. 1) and were 
therefore quite different from the float salinities (Figure 7b). The mapped salinities at the end of the float time series were associated with large mapping errors. Consequently, they had less influence on the final offset estimate, which was now not significantly different from zero. We then ran the modified OW method with $\mathrm{T}$ equal to 5 years (results not shown). Because there were more reference data within $+/-5$ years of the Argo profiles, the differences between the mapped salinities and the float salinities were closer to zero after cycle 80 than if we used $\mathrm{T}$ equal to 2 years. The final offset estimate was quasi-identical to the one obtained with $\mathrm{T}$ equal to 2 years and led to the same conclusion that no correction was necessary. This example illustrates that the choice of the time scale $T$, which is a parameter of the method, and the interpretation of the results should be made in the light of what is known of the variability and what can be resolved by the reference database. It is important to note that the limits of the method are reached when there is not enough reference data within the large spatial scales and within the temporal scale $\mathrm{T}$ for most or all the profiles of the float time series.

We ran the modified OW method using the CTD+Argo reference database for all the 392 unbiased floats found in the North Atlantic. Results are presented for a time scale $\mathrm{T}$ equal to 2 years. The results were not significantly changed if a time scale equal to 5 years was used, meaning that time scales ranging from 2 to 5 years are probably appropriate in this region given the large-scale variability of the salinity field and what can be resolved by the CTD+Argo reference database. The differences between the mapped salinities and the float salinities $\left(\overline{\Delta S_{\text {sta }}}\right)$ for the subset of 392 unbiased Argo floats did not show regional patterns in the Subpolar Region and the Western Subtropical Gyre (Figure 8a), as was the case when the standard OW method was used (Figures $2 \mathrm{a}$ and $2 \mathrm{~b}$ ). Large values of $\overline{\Delta S_{\text {sta }}}$ were still obtained in the eastern North Atlantic, particularly off the coasts of Spain and Portugal. Most of the salinity offsets $\left(\Delta S_{f i t}\right)$ were found within the $[-0.01,+0.01]$ bounds (Figure $8 \mathrm{~b}$ ). The fit errors were larger than those obtained with the original OW method $(60 \%$ of the fit errors were now greater than 0.0025 and even greater than 0.005 in some areas, such as the region off the coasts of Spain and Portugal). Most of the salinity offsets were therefore below the recommended threshold to make an adjustment on float salinity data. These results were consistent with the PI's decisions. Indeed, among the 392 floats, we only found nine floats for which the correction proposed was larger than 0.01 (or smaller than -0.01 ) and more than 
twice the fit error (which should be compared with the 40 floats identified with the original OW method and the CTD+Argo reference database). For four of them (float numbers 6900162, 6900176, 6900515 and 6900614), we think that the delayed mode correction should not have been zero and should be revised. This was later confirmed by the PI, and floats 6900162, 6900176 and 6900515 have already been revised. These floats were excluded from the plots in Figure 8. For the remaining five floats, we do not think the floats need to be corrected for a salinity drift or bias, in accordance with the PI ${ }^{\mathrm{ee}}$ s decision. In fact, for these five floats, the method should be further tuned either by choosing different $\theta$ levels to calibrate the floats or by splitting the float time series when the floats sample very different water masses. For example, for floats that sampled water off the coasts of Spain and Portugal, we suggest choosing $\theta$ levels above the Mediterranean water to calibrate the salinity data.
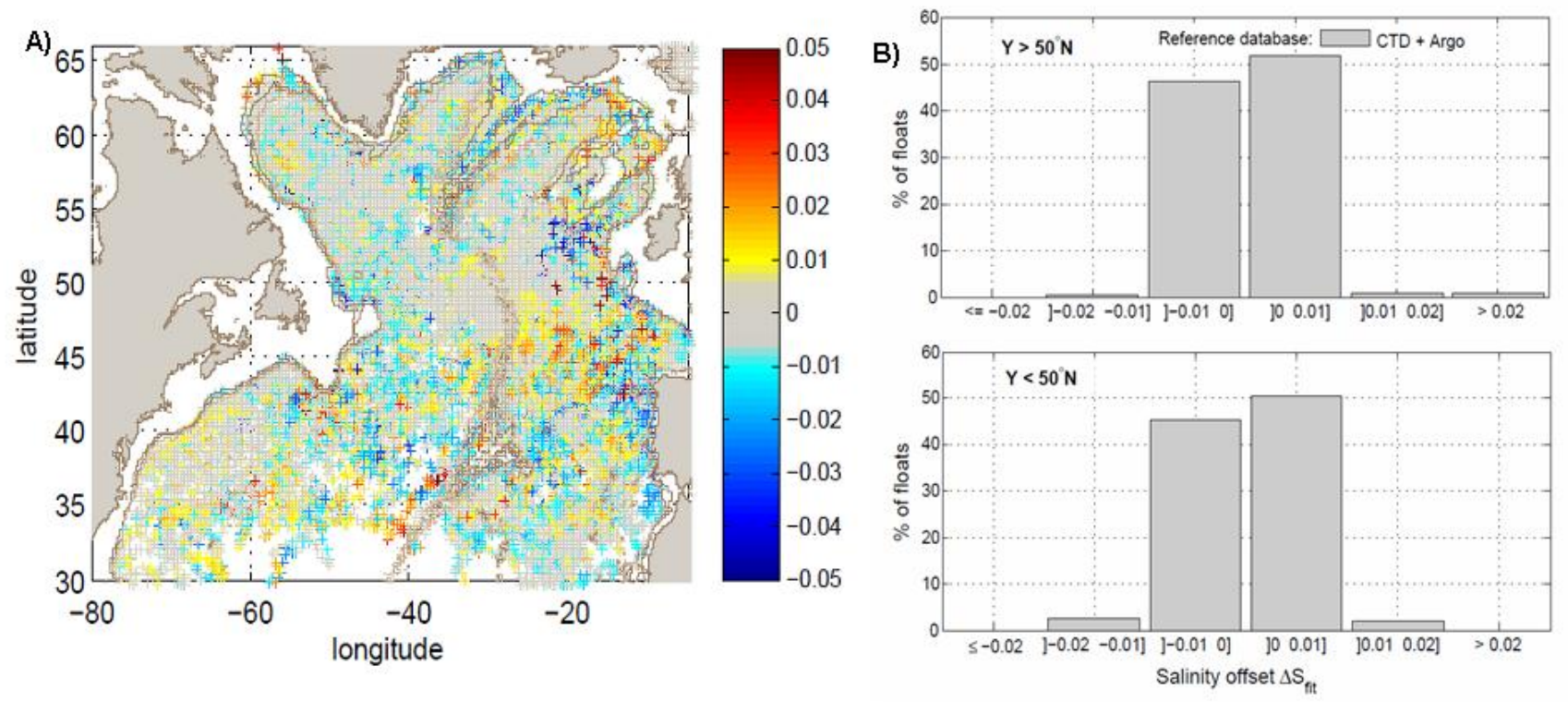

Figure 8: (A) Differences between mapped salinities and float salinities averaged over $10 \theta$ levels of a profile. The modified $O W$ method was used with the CTD + Argo reference database. (B) Distribution of the salinity offsets for the 160 floats found in the North Atlantic, north of $50^{\circ} \mathrm{N}$ (upper panel) and for the 232 floats found south of $50^{\circ} \mathrm{N}$ (lower panel), obtained when the modified $\mathrm{OW}$ method was used with the CTD + Argo reference database.

Finally, we checked the corrections of the 186 floats that were corrected in delayed mode for a salinity offset or drift. We found 22 floats for which the correction applied by the PI differed significantly from the one obtained with our modified OW method and for which we consider it necessary to revise the original correction. Most of these floats were deployed early in the 
Argo programme when very few reference data were available and when the delayed mode adjustment method was still evolving. The list of the 22 floats is given at http://www.argodatamgt.org/Argo-regional-Centers/North-Atlantic-ARC/Overall-

consistency-of-DM-corrections. The PIs or delayed mode operators of the 22 floats were informed and delayed mode corrections have either been revised or are in the process of revision.

\section{Conclusion}

The OW method is widely used and is a powerful way to detect and correct bias in the conductivity sensors of Argo floats. In this study, we proposed modifications of the OW method to account for the large interannual to decadal variability of the large-scale salinity field observed in the North Atlantic. In particular, we modified the covariance function in order to minimize the contribution of the oldest reference data to the large-scale salinity field estimate. The error computation was also modified to obtain more realistic errors for the fit. These modifications concern the mapping error, which now includes errors for the large-scale field estimates as well as the fit error, which now takes into account the lateral dependence between climatological profiles. In the North Atlantic, we found that the modified method generally gave results in accordance with the PI's decision and, in this sense, can be more helpful than the original method for deciding whether the salinity data of a float needs to be corrected or not. The proposed modifications need to be tested in other regions where largescale hydrographic properties vary with time, and further improvements are probably required to better take into account sharp changes in hydrological characteristics and water mass structures, such as those observed in frontal zones.

This study emphasises the need to continue updating the reference database with recent shipboard CTD data to be able to resolve large interannual to decadal variability of the salinity field such as that observed in the North-Atlantic Ocean. Using shipboard CTD data is indeed essential to provide an independent estimation of the salinity field simultaneous to the float measurement and to help detect any systematic error in the Argo data. When a float is processed in delayed mode, it is therefore highly recommended that the results obtained from the OW method when it is run with the CTD+Argo reference database, are cross-validated by the comparison of the float salinity with independent and recent shipboard CTD data, at least for some profiles in the float time series. Ideally, it should be a prerequisite to include an unbiased float in the Argo reference database. This implies that more shipboard CTD data are 
collected close to Argo floats. Süch efforts to maintain an up-to-date shipboard CTD reference database should be conducted in collaboration with shipboard observation communities such as Go-SHIP.

Finally, we used the modified OW method to check the consistency of the Argo salinity dataset available in delayed mode in the North-Atlantic Ocean. We estimated that $1 \%$ (4 out of 392) of the floats with no salinity correction should be adjusted for salinity and that $12 \%$ (22 out of 186) of the floats corrected for a salinity offset or drift should be revised. Overall, salinity corrections need to be reconsidered for $4.5 \%$ of the floats checked in this study. Most of these were deployed in the early period of the Argo programme. This reflects the good overall quality of the Argo database and highlights the progress made by the Argo community for the delayed-mode adjustment of salinity since the beginning of the Argo programme. Consistency checks remain necessary to ensure the overall quality of the Argo dataset. It is planned to perform such checks annually on the North-Atlantic Argo fleet.

\section{Acknowledgements}

We sincerely thank four anonymous reviewers for their reviews, which helped us to improve this manuscript. Cecile Cabanes is funded by CNRS, and Virginie Thierry and Catherine Lagadec are funded by IFREMER. This work is a contribution to the EQUIPEX NAOS (ANR-10- EQPX-40) project funded by the French "Agence Nationale pour la Recherche (ANR) and to the OVIDE and RREX projects supported by IFREMER, CNRS and INSU (Institut National des Sciences de leeUnivers) and by French national programs (LEFE/INSU). OVIDE is a contribution to CLIVAR. Argo data were collected and made freely available by the International Argo Project and the national programmes that contribute to it (http://www.argo.ucsd.edu, http://argo.jcommops.org). Argo is a pilot programme of the Global Ocean Observing System.

\section{Appendix A}

In the large-scale mapping step of the modified OW method, the covariance matrices are determined by the large spatial scales $\left(\mathrm{L}_{\mathrm{x}}\right.$ and $\left.\mathrm{L}_{\mathrm{y}}\right)$, the large cross-isobath scale $(\Phi)$ and by the time scale $\mathrm{T}$ (Table 1).

The data-mapping point covariance matrix $\mathrm{Cdm}$ is then:

$$
C d m_{i o}=V \times \exp \left\{-\left[\frac{\left(x_{i}-x_{o}\right)^{2}}{L_{x}^{2}}+\frac{\left(y_{i}-y_{o}\right)^{2}}{L_{y}^{2}}+\frac{\left(t_{i}-t_{o}\right)^{2}}{T^{2}}+\frac{\left(q_{i}-q_{o}\right)^{2}}{\left(q_{i}^{2}+q_{0}^{2}\right) \Phi^{2}}\right]\right\},
$$


where $\left(x_{i}, y_{i}, t_{i}\right)$ are the position and time of the reference data, $\left(x_{0}, y_{0}, t_{0}\right)$ are the position and time of the float profile. $\mathrm{q}_{\mathrm{i}}$ and $\mathrm{q}_{0}$ are the topographic potential vorticity at the reference data position and the float profile position, respectively. $\mathrm{V}$ is the signal variance of the reference data.

The data-data covariance matrix $C d d$ is:

$C d d_{i j}=V \times \exp \left\{-\left[\frac{\left(x_{i}-x_{j}\right)^{2}}{L_{x}^{2}}+\frac{\left(y_{i}-y_{j}\right)^{2}}{L_{y}^{2}}+\frac{\left(t_{i}-t_{j}\right)^{2}}{T^{2}}+\frac{\left(q_{i}-q_{j}\right)^{2}}{\left(q_{i}^{2}+q_{j}^{2}\right) \Phi^{2}}\right]\right\}+\delta_{i j} E$,

where $\mathrm{E}$ is the noise variance of the reference data computed as in Wong et al., (2003) and $\delta_{i=j}=\left\{\begin{array}{l}1 \text { if } i=j \\ 0 \text { if } i \neq j\end{array}\right.$

\section{References}

Antonov, J. I., R. A. Locarnini, T. P. Boyer, A. V. Mishonov, and H. E. Garcia, 2006. World Ocean Atlas 2005, Volume 2: Salinity. S. Levitus, Ed. NOAA Atlas NESDIS 62, U.S. Government Printing Office, Washington, D.C., 182 pp.

Argo Science Team, 2000. Report of the Argo Science Team $2^{\text {nd }}$ Meeting (AST-2) March 7-9, 2000, Southampton Oceanography Centre, Southampton U.K., http://www.argo.ucsd.edu/iast2.pdf

Barker, P. M., J. R. Dunn, C. M. Domingues, and S. E. Wijffels, 2011: Pressure Sensor Drifts in Argo and Their Impacts, J. Atmos. Ocean. Technol., 28(8), 1036-1049, doi:10.1175/2011JTECHO831.1

Boehme, L. and U. Send, 2005. Objective analyses of hydrographic data for referencing profiling float salinities in highly variable environments. Deep-Sea Res. Part II, 52, 651-664. doi:10.1016/j.dsr2.2004.12.014

Boyer, T.P., J.I. Antonov, H.E. Garcia, D.R. Johnson, R.A. Locarnini, A.V. Mishonov, M.T. Pitcher, O.K. Baranova, I.V. Smolyar, 2006. World Ocean Database 2005. S. Levitus, Ed., NOAA Atlas NESDIS 60, U.S. Government Printing Office, Washington, D.C., 190 pp., DVDs. 
Bretherton, F., R. Davis, and C.Fandry, 1976. A technique for objective analysis and design of oceanic experiments applied to Mode-73. Deep-Sea Res., 23, 559-582. doi:10.1016/00117471(76)90001-2

Gaillard, F., E. Autret, V. Thierry, P. Galaup, C. Coatanoan, and T. Loubrieu, 2009. Quality controls of large Argo datasets. J. Atmos. and Oceanic Tech, 26, 337-351. doi:10.1175/2008JTECHO552.1

Gaillard, F., 2015. ISAS-13-CLIM temperature and salinity gridded climatology. Pôle Océan, doi:10.12770/e23e19d4-dc4d-40d1-8cfd-4e9f70746dd7

Kieke, D., and I. Yashayaev, 2015. Studies of Labrador Sea Water formation and variability in the subpolar North Atlantic in the light of international partnership and collaboration. Prog. Oceanogr., 132, 220-232. doi:10.1016/j.pocean.2014.12.010

Leadbetter, S.J., R.G. Williams, E.L. McDonagh, and B.A. King, 2007. A twenty year reversal in water mass trends in the subtropical North Atlantic. Geophys. Res. Lett., 34, L12608, doi:10.1029/2007GL029957

Ollitrault, M., and J.P. Rannou, 2013. ANDRO: An Argo-Based Deep Displacement Dataset. J. Atmos. Oceanic Technol., 30, 759-788. doi:10.1175/JTECH-D-12-00073.1

Owens, W.B. and A.P.S. Wong, 2009. An improved calibration method for the drift of the conductivity sensor on autonomous CTD profiling floats by $\Theta-S$ climatology. Deep-Sea Res. Part I, 56, 450-457. doi:10.1016/j.dsr.2008.09.008

Roemmich, D., 1983. Optimal Estimation of Hydrographic station data and derived fields. J. Phys. Oceanogr., 13, 1544-1549. doi:10.1175/1520-0485(1983)013<1544:OEOHSD $>$ 2.0.CO;2

Sarafanov, A., A. Sokov, A. Demidov, and A. Falina, 2007. Warming and salinification of intermediate and deep waters in the Irminger Sea and Iceland Basin in 1997-2006. Geophysical Research Letters, 34, L23609, doi:10.1029/2007GL031074.

Willis, J. K., J. M. Lyman, G. C. Johnson, and J. Gilson, 2007. Correction to "Recent cooling of the upper ocean”, Geophys. Res. Lett., 34, L16601, doi:10.1029/2007GL030323.

Wong, A.L.S., J.M. Johnson, and W.B. Owens, 2003. Delayed-Mode Calibration of Autonomous CTD Profiling Float Salinity Data by $\theta-S$ Climatology. J. Atmos. and Oceanic Tech., 20,308-318, doi:10.1175/1520-0426(2003)020<0308:DMCOAC >2.0.CO;2 
Wong, A.L.S, R. Keeley, T. Carval, and the Argo Data Management Team, 2014. Argo

quality control manual, Version 2.9.1, 56pp,

http://archimer.ifremer.fr/doc/00228/33951/32470.pdf

Highlights

- Method used to detect bias in Argo float conductivity sensors is improved

- Temporal variability of the large-scale salinity field is taken into account

- Computation of the errors for salinity data corrections is improved

- Consistency of the salinity data corrections in the North Atlantic is checked 